International Journal of Modern Physics C Vol. 25, No. 7 (2014) 1450025 (12 pages)

(c) The Authors

DOI: $10.1142 /$ S0129183114500259

\title{
Effects of aspiration-induced adaptation and migration on the evolution of cooperation
}

\author{
Yu Peng \\ School of Science, Southwest University of Science and Technology \\ Mianyang, 621010/Sichuan, P. R. China \\ pengyu@swust.edu.cn \\ Xu-Wen Wang* \\ Department of Modern Physics \\ University of the Science and Technology of China \\ Hefei, 230026/Anhui, P. R. China \\ xuwenwang86@gmail.com \\ Qian Lu \\ School of Science \\ Southwest University of Science and Technology \\ Mianyang, 621010/Sichuan, P. R. China \\ Qing-Ke Zeng \\ Department of Modern Physics \\ University of the Science and Technology of China \\ Hefei, 230026/Anhui, P. R. China \\ Bing-Hong Wang* \\ Department of Modern Physics \\ University of the Science and Technology of China \\ Hefei, 230026/Anhui, P. R. China \\ College of Physics and Electronic Information Engineering \\ Wenzhou University \\ Wenzhou, 325035/Zhejiang, P. R. China \\ The Research Center for Complex System Science \\ University of Shanghai for Science and Technology \\ and Shanghai Academy of System Science \\ Shanghai 200093, P. R. China \\ bhwang@ustc.edu.cn \\ Received 4 November 2013 \\ Accepted 7 January 2014 \\ Published 10 February 2014
}

*Corresponding authors. 


\section{Y. Peng et al.}

In the light of the prospect theory (PT), we study the prisoner's dilemma game (PDG) on square lattice by integrating the deterministic and Data envelopment analysis (DEA) efficient rule into adaptive rules: the individual will change evolutionary rule and migrate if its payoff is lower than their aspiration levels. Whether the individual choose to change the evolutionary rule and migrate is determined by the relation between its payoff and aspiration level. The results show that the cooperation frequency can hold unchange with the increasing of temptation to defect. The individual chooses to adopt DEA efficient rule and to migrate that can induce the emergence of cooperation as the payoff is lower than its aspiration.

Keywords: Adaptive evolutionary rule; migration; prospect theory; data envelopment analysis efficient rule; evolutionary game.

PACS Nos.: 02.50.Le, 87.23.Ge, 89.75.Fb.

\section{Introduction}

Cooperation is fundamental to biological and social systems. ${ }^{1}$ Evolutionary games have become an important tool to study the emergence of cooperative behavior in a competitive setting. ${ }^{2,3}$ It has been attracted more attention to understand the emergence and persistence of cooperation among selfish individuals. Recent researches are trying to combine the evolutionary cooperative behaviors with the spatial structures. $^{4-9}$ The more important mechanism have been introduced to study the evolutionary games on complex networks. ${ }^{8-12}$ It is well accepted that the evolutionary rule plays an important role in the evolution of cooperation, and individuals can update their strategies by adopting different rules in the evolutionary games. The deterministic rule be proposed by Nowak and May, ${ }^{5}$ the stochastic evolutionary rule be introduced by Szabó and Töke ${ }^{4}$ are used commonly. Apart from these, learning theory has provided a reasonable framework to study the evolutionary games, since individuals could be viewed as adaptive agents, hence, they can adopt a self-questioning mechanism ${ }^{13-15,53}$ and assess the relative efficiency of the strategy, via data envelopment analysis (DEA). ${ }^{16}$ Moreover, individuals can update their strategies according to the difference between the actual and aspiration payoff based on learning theory. ${ }^{17}$ As to the modeling of cooperation among different species with different cognitive abilities, Nowak reviewed the evolution of cooperation from different disciplines. ${ }^{18}$ Szabó and Fáth reviewed evolutionary games on graphs. ${ }^{19}$ In biological models, individuals simply update their strategies, e.g. death-birth rule or birth-death rule. ${ }^{20}$

Many important mechanisms have been introduced to study the cooperative behaviors, such as costly punishment, ${ }^{21,22}$ reputation, ${ }^{23,24}$ and social diversity. ${ }^{25-27}$ As is well-known, migration has a significant impact on evolutionary games. ${ }^{28-35,54,55}$ Generally, two different types of migrations have been studied. One type is the movement for individuals from original sites to other sites on a spatial lattice, which is also referred to as mobility. ${ }^{30,31,36-38}$ Reichenabach et al. ${ }^{36}$ indicated that biodiversity can be promoted, when the parameter "mobility" (which measures the capability of individuals to jump to an adjacent empty site) below some critical threshold. Another type of migration occurs between distinct groups, which can be 
the interior and the exterior of the population, or among subpopulations. ${ }^{38}$ The aspirations have the important role in evolutionary games, ${ }^{39-41}$ Recently, Yang et al. have proposed an aspiration-induced migration in which individuals migrate to new sites, while their payoff are below some aspiration levels. ${ }^{34}$ The universal role of migration in the evolution of cooperation has been discussed in Ref. 34 .

The prospect theory $(\mathrm{PT})^{42}$ is an alternative way to the expected utility theory (EUT) ${ }^{43,44}$ The basic idea of the PT is that the alternatives are evaluated by a different function in terms of gains and losses with respect to a reference point. The function is suggested to be concave for gains and convex for losses and steeper for losses than for gains. In human models, individuals have bounded rationality, they have sufficient knowledge and cognitive skill to learn from others, they can select their best-response or better-response strategies according to others' strategies. ${ }^{45,46}$ In the light of the PT, individuals will change evolutionary rules and migrate to new sites, if their current selections are unsatisfactory with aspirations.

Previous works only adopt one type evolutionary rule regardless of the payoff level. From the PT, we know that individuals would like to adopt different evolutionary rule at different payoff level. In this paper, we introduce the aspirationinduced adaptive evolutionary rule and migration $^{34}$ to study the evolution of cooperation in human social systems. An individual will change the evolutionary rule, or move to another empty site, if its current payoff is lower than the aspiration level. Here, the aspiration level can be understood as the extent of satisfaction of individuals relative to its neighbors. Individuals can perform an adaptive movement with adaptive rule in order to gain more satisfactory payoff.

\section{PT and Adaptive Evolutionary Rule}

\subsection{Some conclusions of the PT}

The PT was first formulated by Kahneman and Tversky as an alternative method of explaining choices made by individuals under conditions of risk. Kahneman was awarded the 2002 Nobel Memorial Prize in Economics for his work in the PT. The PT was designed, in essence, as a substitute for the EUT. Kahneman and Tversky realized the fact that the EUT model did not fully describe the manner in which individuals made decisions in risky situations and that therefore, there were instances in which a decision-maker's choice could not be predicted. For example, they pointed out that the EUT did not explain the manner in which framing could change the decision of the individual, nor did it explain why individuals exhibited risk-seeking behavior in some instances and risk-averse behavior in others. In order to compensate these characteristics of individual behavior which are unexplained by the EUT, Kahneman and Tversky developed a new choice model and called it the PT. In the EUT, decisions in risky situations are made according to final wealth and probabilities. Whereas in the PT, these decisions are made based on values assigned to gains and losses with respect to a reference point and decision weights. They pointed 
out that the over weighting of low probabilities might explain why individuals made a choice to accept insurance and gambling at the same time.

Kahneman and Tversky applied the psychophysical principles of evaluation to examine the effect of framing and the violation of the principle of invariance that underlies the rational theory of choice. They found out more evidence that individuals make decisions based on changes in wealth rather than wealth in the final state. ${ }^{47}$ They argued that the choices that individuals made in risky situations exhibited several characteristics. It brings about risk-aversion in choices involving certain gains and risk-seeking in choices involving certain losses.

\subsection{DEA efficient rule}

$\mathrm{DEA}^{17}$ is one of the most widely accepted methods to evaluate the relative efficiency of a homogeneous group of decision making units (DMUs) based on observed data. DEA has extended the engineering efficiency of units with single input and single output. It has a strong background in engineering and technology, and has many advantages in avoiding subjective factors, reducing errors, use of simple arithmetic and so on. Since the advent of DEA, there has been an impressive growth in both theoretical developments and applications of the ideas to practical situations. ${ }^{48}$ In evolvement game, each individual interacts only with its neighbors to gain the payoff by summing up all its encounters. Individuals pay close attention to the payoff of the same-strategy neighbors. They assess their strategy by DEA method ${ }^{16}$ and obtain the relative efficiency value (REV) of the strategy selected in the round. The REV of the strategy is defined as the fraction whose numerator is its payoff, and whose denominator is the highest payoff of the same-strategy neighbors (including itself). Each individual chooses its best strategy based on REVs in that round. The DEA efficient rule to update strategy is stated as follows.

(i) If $\mathrm{REV}=1$, its strategy is called DEA efficient, it will persist in its selection.

(ii) If REV $<1$, its strategy is called DEA inefficient, it will adopt another strategy to play a virtual game with all its neighbors, and calculate the virtual payoff, to gain the virtual REVs. Comparing the virtual REVs with the actual REV, each individual can get its optimal strategy corresponding to the highest REV. In the next round game, it will choose the optimal strategy.

(iii) All individuals update their strategy simultaneously.

It is worth mentioning that being DEA efficient is equivalent to being Pareto efficient for a DMU. ${ }^{49}$

\subsection{The adaptive evolutionary rule}

To gain more satisfactory payoff, individuals usually adjust the evolutionary rule, according to the aspiration level which is relative to neighbors. Being extracted from our daily experiences and the PT, the adaptive evolutionary rule is stated as follows. 
The aspiration level is discrete by using thresholds, the interval between two consecutive thresholds is called aspiration interval. Comparing the actual payoff with the aspiration level, individual adopts different evolutionary rule responding to different aspiration interval. All the evolutionary rules with the corresponding intervals are integrated into one rule called the adaptive evolutionary rule. With the aspiration intervals and the evolutionary rules in different combination sequence, many adaptive evolutionary rules could be integrated.

For a simple case, there are two aspiration intervals and two evolutionary rules, from the point of the PT, one rule may be risk-aversion and the other rule may be risk-seeking especially. We consider the DEA efficient rule ${ }^{16}$ which can induce the steady and high cooperation frequency, and the most commonly used deterministic rule. ${ }^{5}$ The deterministic rule is also called the best-takes-over rule, i.e. each individual compares its payoff with that of its neighbors and updates its strategy by the following one (including itself) with the greatest payoff. To some extent, the DEA efficient rule could be seen as one of the risk-aversion rules for updating strategy based on the relative efficient value, and the deterministic rule could be seen as one of the risk-seeking rules for updating strategy by imitating the strategy from the one with the highest payoff among itself and its immediate neighbors. Of course, other evolutionary rules could be chosen as well.

The above two evolutionary rules can be integrated into two adaptive rules:

Adaptive Rule 1: Comparing the actual payoff with the aspiration level, each individual will choose the deterministic rule in the next round game if the actual payoff below its aspiration level, otherwise, it will choose the DEA efficient rule.

Adaptive Rule 2: Comparing the actual payoff with the aspiration level, each individual will choose the deterministic rule in the next round game if the actual payoff reaches or exceeds its aspiration level, otherwise, it will choose the DEA efficient rule.

\section{The Model and Simulations}

\subsection{The aspiration level for migration and integration}

Following the previous studies, ${ }^{34,50}$ the migration aspiration level $P_{i}$ for individual $i$ to move is defined as $P_{i}=k_{i} B$, where $k_{i}$ is the number of neighbors of individual $i$ and $B$ is a control parameter ( $B$ is same for all individuals). This definition is based on the following consideration: maintaining a social contact is usually costly. ${ }^{51}$ For simplicity, we assume that an individual cost $B$ to maintain the link with one of its neighbors, then $P_{i}$ is the total cost to maintain social links with all its neighbors for individual $i$. We call $P_{i}$ the migration threshold as well.

Similarly, the integration aspiration level $Q_{i}$ for individual $i$ to change evolutionary rule is defined as $Q_{i}=k_{i} A$, where $k_{i}$ is the number of neighbors of individual $i$ and $A$ is a control parameter ( $A$ is same for all individuals). This definition is based on the following consideration: the expected payoffs from one of individual's neighbor 
is $A$, while the aspiration level have not been reached, one may try to maximize its payoffs through adopting different evolutionary rule. The integration aspiration level $Q_{i}$ is the total mentality critical value of payoffs for individual $i$ to persist in current evolutionary rule, it is also the threshold to divide aspiration level into intervals responding to different evolutionary rules, respectively.

\subsection{The model}

We consider the prisoner's dilemma game $(\mathrm{PDG})^{52}$ in our research. In principle, the PDG is a game played by two players, each of whom chooses one of two strategies, cooperation or defection. They both receive payoff $R$ upon mutual cooperation and $P$ upon mutual defection. If one defects while the other cooperates, the cooperator receives $S$ while the defector gets $T$. The ranking of the four payoff values is $T>R>P>S$. Thus, in a single round of the PDG, it is best to defect regardless of the opponent's decision. The PDG has attracted much attention in theoretical and experimental studies of cooperative behavior. Following common practice, ${ }^{5}$ we set $T=b(1<b<2), R=1$ and $P=S=0$, where $b$ is the only payoff parameter representing the temptation to defect.

The PDG individuals are situated on a square lattice of $L \times L$ sites with periodic boundary conditions, each site is either empty or occupied by one individual with equal probability. Initially, each individual is designated either as a cooperator or defector with equal probability. At each time-step, individual $i$ plays the PDG game with its directed neighbors, its payoff is the sum of all the payoffs acquired from its neighbors. Then, by comparing the payoff with its aspiration level, individual $i$ will stay in current site if the payoff reaches or exceeds its aspiration level, otherwise, it will move to a randomly selected site. Finally, individual $i$ updates its strategy by adaptive evolutionary rule which is also chosen by comparing the payoff with the integration aspiration level. To avoid isolated case, we assume that an isolated individual makes mandatory movement. For all the simulations, individuals update their strategies synchronously.

\subsection{Simulation results on regular lattices}

Simulations are carried out on a square lattice with size $N=100 \times 100$ and $50 \%$ empty sites, the individuals are assigned on sites randomly. Each individual compares its actual payoff with the aspiration level, then it decides whether to leave or stay in its current site.

First, we investigate the frequency of cooperators as functions of temptation to defect $b$ and the integration aspiration parameter $A$ for Adaptive Rule 1. As shown in Fig. 1(a), as $B=0$, the individuals except isolated ones will not migrate to other sites. In the situation of half empty sites, generally, the payoffs of cooperators and their neighbors who also adopt cooperation strategy are higher than their aspiration levels for smaller values of $A$, so the DEA efficient rule is used for them. According to DEA method, the REV is calculated by comparing their own payoff with the highest 


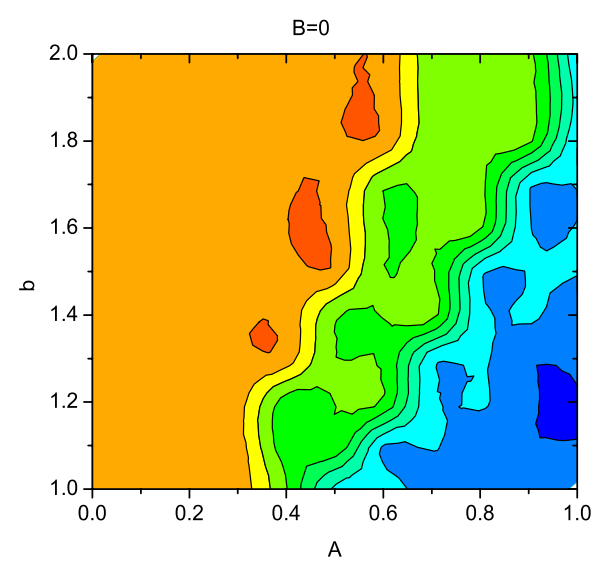

(a)

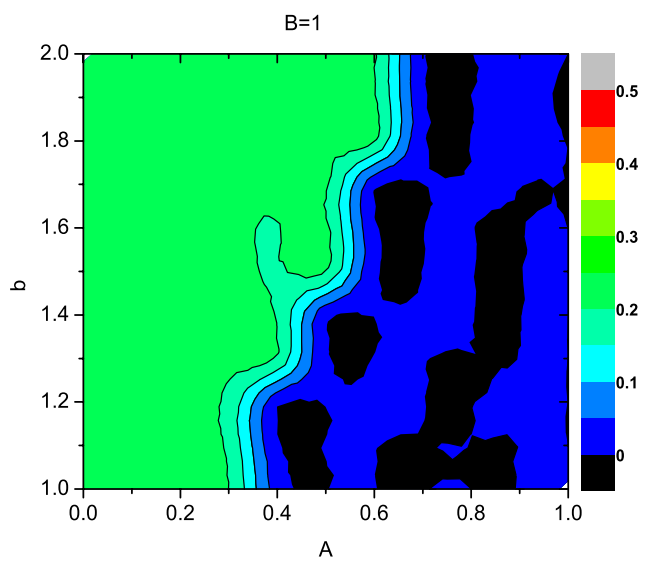

(b)

Fig. 1. (Color online) Fraction of cooperators as functions of the temptation to defect $b$ and the aspiration level $A$. The evolutionary rule is the Adaptive Rule 1. (a) $B=0$; (b) $B=1$. The simulation results are averaged by last 1000 time-steps of a total of 10000 time-steps of evolution. Each data point results is obtained by averaging over 20 different initial states.

payoff of its neighbor who adopts the same strategy with itself, ${ }^{16}$ which leads to the frequency of cooperators maintained at a certain value as the $b$ increases.

To further study the effect of DEA efficient rule on the frequency of cooperators, we assume the number of $C$ neighbors of individual $i$ to be $n$, then, the payoff of individual $i$ with strategy $X(X=C$ or $X=D)$ is

$$
R(X)= \begin{cases}n & X=C, \\ n b & X=D .\end{cases}
$$

To calculate the relative efficiency of individual $i$ with strategy $X$, we denote $n^{\prime}$ the number of $C$ neighbors of the individual with the highest payoff among its samestrategy neighbors (including itself). Specially, as $k_{i}=4$, the REV of strategy $X$ for individual $i$ is shown in Table $1 .^{16}$

From Table 1, we know that the REV has nothing to do with the parameter $b$, which induces the frequency of cooperators can hold unchanged with the increasing $b$.

Table 1. REV of strategy $X$ for individual $i$.

\begin{tabular}{ccccccccc}
\hline & \multicolumn{9}{c}{$X=C$} & & \multicolumn{3}{c}{$X=D$} \\
\cline { 2 - 5 } \cline { 6 - 8 } & $n^{\prime}=1$ & $n^{\prime}=2$ & $n^{\prime}=3$ & $n^{\prime}=4$ & & $n^{\prime}=1$ & $n^{\prime}=2$ & $n^{\prime}=3$ \\
\hline$n=0$ & 1 & 1 & 1 & 1 & - & - & - \\
$n=1$ & 1 & $1 / 2$ & $1 / 3$ & $1 / 4$ & & 1 & $1 / 2$ & $1 / 3$ \\
$n=2$ & 1 & 1 & $2 / 3$ & $1 / 2$ & & 1 & $2 / 3$ \\
$n=3$ & 1 & 1 & 1 & $3 / 4$ & 1 & 1 & 1 \\
$n=4$ & 1 & 1 & 1 & 1 & & 1 & 1 \\
\hline
\end{tabular}




\section{Y. Peng et al.}

For the larger integration aspiration levels, most of individuals' payoffs are lower than their aspirations level, the ratio of individuals who adopt DEA efficient rule decreases with the $A$, however, the deterministic rule induces individuals to defect, the frequency of cooperators is lower than the case of small integration aspiration level. As shown in Fig. 1(a), the frequency of cooperators almost be a constant of 0.4 , which is less than the result in Ref. 14. Since the number of neighbors for individual is less than 4. It is known that the frequency of cooperators increases with the number of neighbors at the fixed payoff parameter. So, the DEA rule can efficiently prevent the spreading of defection behaviors. ${ }^{16}$

As for as the migration effect is considered, as shown in Fig. 1(b), the change of frequency of cooperators as functions of $b$ and aspiration parameter $A$ when $B=1$ is similar to the case of without migration. Overall, the migration does not contribute to the cooperation behavior. The migration aspiration makes the individuals whose payoffs are lower than aspiration levels to occupy the other empty sites, then, less individuals can get the payoffs higher than aspiration than the case without migration, so, the fraction of DEA efficient rule users is less than the case of $B=0$. On contrary, the Adaptive Rule 2 is more conductive to the emergence of cooperation than Adaptive Rule 1 which is shown in Fig. 2. A larger integration aspiration level induces the majority of individuals to adopt DEA strategy, consequently, the frequency of cooperators can hold on a steady and high level with the increasing of parameter $b$ and the aspiration level $A$. It can be seen from the Fig. 2(b), while the individuals can migrate to empty sites, there exists a optimal range of the integration aspiration level, in which the cooperation level is higher. An important result from Adaptive Rule 2 is that the migration leads to higher cooperation level.

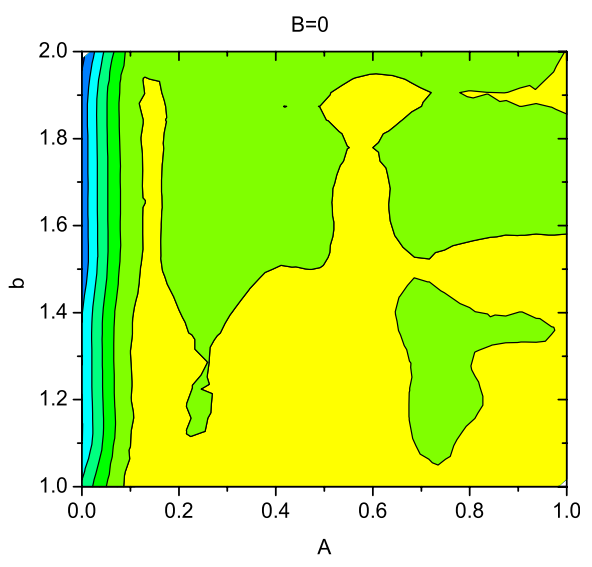

(a)

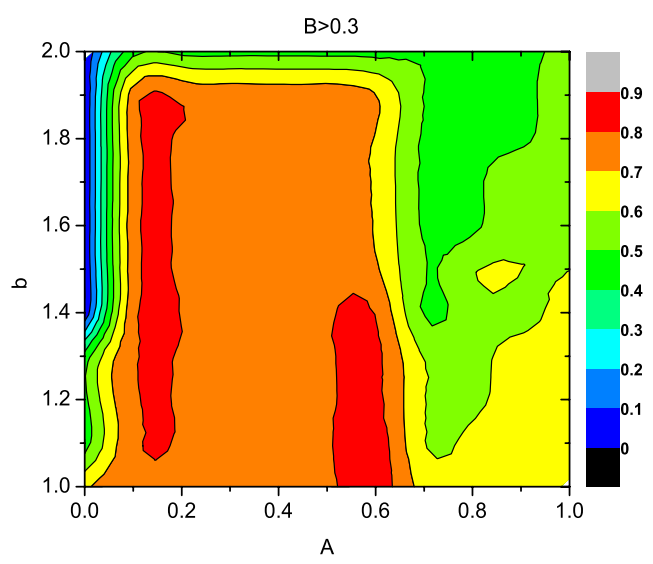

(b)

Fig. 2. (Color online) Fraction of cooperators as functions of the temptation to defect $b$ and the aspiration level $A$. The evolutionary rule is the Adaptive Rule 2. (a) $B=0$; (b) $B>0.3$. The simulation results are averaged by last 1000 time-steps of a total of 10000 time-steps of evolution. Each data point results is obtained by averaging over 20 different initial states. 
From the above discussion, it is obvious that the frequency of cooperators is mainly determined by the fraction of individuals who adopt the DEA strategy. To gain the intuitive understanding of the evolution in the system, we investigate the ratio of individuals adopting the DEA efficient rule to the total population with the temptation to defect $b$, the evolutionary rule is the Adaptive Rule 1 and without migration. Given a fixed aspiration level, the number of the DEA efficient rule users increases with the temptation to defect $b$. Since the DEA efficient rule can produce a higher and steady cooperation frequency, $f_{C}$ will be nondecreasing with the increasing of $b$. Figure 3(b) also shows that the ratio of the DEA efficient rule users is increasing with the decreasing of the values of $A$, which is in agreement with the results in Fig. 1(a).

Whether or not a individual choose DEA strategy is determined by the relation between aspiration level and its payoff, then, it is necessary to investigate the average payoff for each individual. From the former discussion, we know that the average payoff of individual $i$ is

$$
R_{i}=f_{C} R_{i}(C)+\left(1-f_{C}\right) R_{i}(D)=k_{i} f_{C}^{2}+k_{i}\left(1-f_{C}\right) f_{C} b .
$$

Comparing the average payoff $R_{i}$ with the integration aspiration level $Q_{i}=k_{i} A$, we know, when $f_{C}^{2}+\left(1-f_{C}\right) f_{C} b>A$, the individual $i$ will adopt the DEA efficient rule according to the Adaptive Rule 1 . Here, $f_{C}$ is associated with $b, A$ and $B$. For the fixed values of $A$ and $B$, the expression $f_{C}^{2}+\left(1-f_{C}\right) f_{C} b$ could be seen as a function of the temptation to defect $b$. Using the simulation results in Fig. 1(a), the curves described by the function $f_{C}^{2}+\left(1-f_{C}\right) f_{C} b$ are similar to Fig. 3 . One can see from Fig. 3 that the ratio of the DEA efficient rule users exhibits a step-like structure with varying $b$. The curve of the function $f_{C}^{2}+\left(1-f_{C}\right) f_{C} b$ and the curve of the ratio of the DEA efficient rule users have the similar step structure with the fixed values of $A$ and $B$.

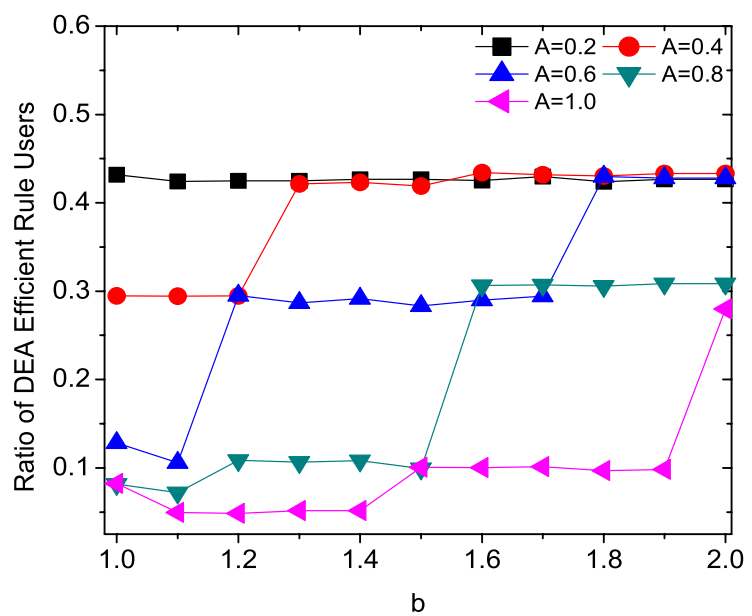

Fig. 3. (Color online) The ratio of the DEA efficient rule users to the total population as a function of the temptation to defect $b$ and the evolutionary rule is the Adaptive Rule 1 as $B=0$. 
Obviously, the size of empty sites can influence the number of neighbors for an individual. With the condition of half empty sites, only the case that the individual $i$ adopt is cooperation strategy and its neighbors are also cooperators, the payoff of individual $i$ is higher than the aspiration level, while the defectors are not willing to adopt DEA strategy. Hence, the ratio of the DEA efficient rule users is approximately equal to the frequency of cooperators. We can see that the result of Fig. 3 is in agreement with result of Fig. 1(a).

It has been known that the DEA efficient rule produces a steady and high cooperation frequency in spatial games. ${ }^{16}$ When the evolutionary rule is the Adaptive Rule 1, for low integrate aspiration level, most individuals adopt the DEA efficient rule. Consequently, the cooperation frequency will keep up a high level in the stationary state. On the contrary, for high integrate aspiration level, most individuals adopt the deterministic rule. For moderate aspiration level, on the one hand, the number of the DEA efficient rule users accounts for high proportion of the population, and on the other hand the cooperators of deterministic rule users can form stable clusters since high benefits of mutual cooperation ensure them to stay in cooperator clusters, and the defectors of deterministic rule users avoid gathering together because the payoffs of mutual defection are low. ${ }^{34}$ As a result, the cooperation frequency decreases as the integrate aspiration level increases.

\section{Conclusion}

In summary, we have incorporated aspiration-induced adaptive evolutionary rule and migration mechanism in the evolutionary PDG on square lattice. In the light of the PT, an individual will alter evolutionary rule and migrate, if its payoff is lower than the aspiration level. The results show that there exist optimal ranges of the integration aspiration level and the temptation to defect, which can lead to the higher frequency of cooperators by some special migrate aspiration level. We find that for some fixed integration and migration aspiration level, the cooperation frequency is nondecreasing with the increasing of the temptation to defect by using the Adaptive Rule 1. The individual chooses to adopt DEA efficient rule and to migrate can induce the emergence of cooperation as the payoff is lower than its aspiration. Furthermore, the mechanism of the adaptive evolutionary rule may mix other evolutionary rules corresponding to different aspiration levels. A similar method can be introduced on other networks, and a similar discussion can be carried out for other expressions of aspiration level.

\section{Acknowledgments}

B.-H. W. was supported by NSFC under Grant Nos. 11275186 and 91024026, and the Major Important Project Fund for Anhui University Nature Science under Grant No. KJ2011ZD07. 


\section{References}

1. A. M. Colman, Game Theory and Its Applications in the Social and Biological Sciences (Butterworth-Heinemann, Oxford, 1995).

2. J. M. Smith, Evolution and the Theory of Games (Cambridge University Press, Cambridge, UK, 1982).

3. H. Gintis, Game Theory Evolving (Princeton University Press, Princeton, NJ, 2000).

4. G. Szabó and C. Töke, Phys. Rev. E 58, 69 (1998).

5. M. Nowak and R. M. May, Nature 359, 826 (1992).

6. C. Hauert and M. Doebeli, Nature 428, 643 (2004).

7. G. Szabó and C. Hauert, Phys. Rev. Lett. 89, 118101 (2002).

8. G. Szabó and C. Hauert, Phys. Rev. E 66, 062903 (2002).

9. F. C. Santos and J. M. Pacheco, Phys. Rev. Lett. 95, 098104 (2005).

10. G. Szabó and J. Vukov, Phys. Rev. E 69, 036107 (2004).

11. X.-W. Wang, H.-F. Zhang, S. Nie and B.-H. Wang, Physica A, Stat. Mech. Appl. 392, 4668 (2013).

12. J. Vukov and G. Szabó, Phys. Rev. E 71, 036133 (2005).

13. W.-X. Wang, J. Ren, G.-R. Chen and B.-H. Wang, Phys. Rev. E 74, 056113 (2006).

14. K. Gao, W.-X. Wang and B.-H. Wang, Physica A, Stat. Mech. Appl. 380, 528 (2007).

15. M. Perc and A. Szolnoki, BioSystems 99, 109 (2010).

16. Y. Peng, B.-H. Wang, Q.-K. Zeng, Q. Lu and H. Wang, Physica A, Stat. Mech. Appl. 391, 2000 (2012).

17. A. Charnes, W. W. Cooper and E. Rhodes, Euro. J. Oper. Res. 2, 429 (1978).

18. M. Nowak, Science 314, 1560 (2006).

19. G. Szabó and G. Fáth, Phys. Rep. 446, 97 (2007).

20. H. Ohtsuki, C. Hauert, E. Lieberman and M. Nowak, Nature 441, 502 (2006).

21. C. Hauert, A. Traulsen, H. Brandt, M. A. Nowak and K. Sigmund, Science 316, 1905 (2007).

22. H. Ohtsuki, Y. Iwasa and M. A. Nowak, Nature 457, 79 (2009).

23. M. A. Nowak and K. Sigmund, Nature 393, 573 (1998).

24. H. Ohtsuki and Y. Iwasa, J. Theor. Biol. 231, 107 (2004).

25. F. C. Santos, M. D. Santos and J. M. Pacheco, Nature 454, 213 (2008).

26. M. Perc and A. Szolnoki, Phys. Rev. E 77, 011904 (2008).

27. H.-X. Yang, W.-X. Wang, Z.-X. Wu, Y.-C. Lai and B.-H. Wang, Phys. Rev. E 79, 056107 (2009).

28. M. Enquist and O. Leimar, Anim. Behav. 45, 747 (1993).

29. C. A. Aktipis, J. Theor. Biol. 231, 249 (2004).

30. M. H. Vainstein, A. T. C. Silva and J. J. Arenzon, J. Theor. Biol. 244, 722 (2007).

31. D. Helbing and W. Yu, Proc. Nat. Acad. Sci. USA 106, 3680 (2009).

32. E. A. Sicardi, H. Fort, M. H. Vainstein and J. J. Arenzon, J. Theor. Biol. 256, 240 (2009).

33. S. Meloni, A. Buscarino, L. Fortuna, M. Frasca, J. Gómez-Gardeñes, V. Latora and Y. Moreno, Phys. Rev. E 79, 067101 (2009).

34. H.-X. Yang, Z.-X. Wu and B.-H. Wang, Phys. Rev. E 81, 065101 (2010).

35. H.-X. Yang and B.-H. Wang, Chinese Sci. Bull. 56, 3693 (2011).

36. T. Reichenbach, M. Mobilia and E. Frey, Nature 448, 1046 (2007).

37. T. Reichenbach, M. Mobilia and E. Frey, J. Theor. Biol. 256, 368 (2008).

38. R. Boyd and P. J. Richerson, J. Theor. Biol. 257, 331 (2009).

39. M. Perc and Z. Wang, PLoS ONE 5, e15117 (2010).

40. Z. Wang and M. Perc, Phys. Rev. E 82, 021115 (2010).

41. M. Perc, Phys. Rev. E 84, 037102 (2011).

42. D. Kahneman and A. Tversky, Econometrica 47, 263 (1979). 
43. J. Von Nueman and O. Morgenstern, Theory of Games and Economic Behavior (Princeton University Press, Princeton, New Jersey, 1947).

44. K. D. Edwards, Int. Rev. Financ. Anal. 5, 19 (1996).

45. E. Hopkins, Games Econ. Behav. 29, 138 (1999).

46. L. Blume, Games Econ. Behav. 11, 111 (1995).

47. A. Tversky and D. Kahneman, J. Bus. 59, 251 (1986).

48. W. D. Cook and L. M. Seiford, Euro. J. Oper. Res. 192, 1 (2009).

49. A. Charnes, W. W. Cooper, B. Golany, L. M. Seiford and J. Stutz, J. Econ. 30, 91 (1985).

50. X. Chen and L. Wang, Phys. Rev. E 77, 017103 (2008).

51. N. Masuda, Proc. R. Soc. B, Biol. Sci. 274, 1815 (2007).

52. R. Axelrod and W. D. Hamilton, Science 211, 1390 (1981).

53. M. W. Macy and A. Flache, Proc. Natl. Acad. Sci. USA 99, 7229 (2002).

54. X. Chen, A. Szolnoki and M. Perc, Phys. Rev. E 86, 036101 (2012).

55. X. Chen, A. Szolnoki and M. Perc, Europhys. Lett. 99, 68003 (2012). 